\title{
Visualising the Three-dimensional Morphology and Surface Structure of Metallic Nanoparticles at Atomic Resolution by Automated HAADF STEM Atom Counting
}

\author{
Lewys Jones ${ }^{1}$, Vidar T. Fauske ${ }^{2}$, Katherine E. MacArthur ${ }^{1}$, Antonius T. J. van Helvoort ${ }^{2}$, and \\ Peter D. Nellist ${ }^{1}$ \\ 1. Department of Materials, University of Oxford, Oxford, UK. \\ 2. Department of Physics, Norwegian University of Science and Technology, Trondheim, Norway.
}

Because of their large proportion of surface atoms and favourable chemical activity, metallic nanoparticles are used to catalyse a wide range of technologically important reactions [1]. However, many industrial catalysts utilise expensive and/or rare metals, leading to the desire to account for their content and efficiency at the atomic scale. High-angle annular dark-field scanning transmission electron microscopy (HAADF STEM) proves a powerful tool here, as this readily interpretable incoherent imaging mode shows direct mass-thickness contrast. Using aberration correctors such dark-field data can be recorded at atomic resolution and subsequently analysed on an atomic column by column basis.

In this work, pure platinum nanoparticles were imaged resulting in contrast that depends on the thickness of individual atomic columns. High-resolution images were recorded using a JEOL-ARM200F operated at $200 \mathrm{kV}$ where convergence and detector angles were carefully measured as $27 \mathrm{mrad}$ and 69 $-279 \mathrm{mrad}$ respectively. The efficiency of the annular detector was also calibrated to allow the data to be expressed in units of 'fractional beam-current' [2]. Where necessary images were compensated for stage drift using the Jitterbug plug-in for Digital Micrograph from HREM Research, followed by magnification calibration using the images' Fourier transforms [3]. Other than this the data were not smoothed or filtered in any way. After magnification calibration, the raw data (Figure 1, left) and the detector efficiency scan were passed to the in-house 'Absolute Integrator' software [4]. This software automatically identifies the image peak-positions, normalises the data to units of fractional beam-current (accounting for detector efficiency as well as amplifier black-level and gain), performs a locally adaptive background subtraction (to account for the amorphous carbon-black support), divides the image into Voronoi polygons and integrates the signal at each atomic column to yield a map of the absolute scattering cross-sections (Figure 1, right) [5]. The quantification procedure is both user-friendly and efficient, requiring around one minute on a standard desktop computer.

Comparing the measured scattering cross-sections with simulation (multi-slice, 30 phonon runs), the number of atoms per column was identified. Using these assignments, a provisional three-dimensional (3D) model was built using the lateral ( $x-y)$ positions from the peak-finding stage, though the absolute $\mathrm{z}$-coordinates are not determined. Owing to the beam-sensitivity of the particles and the desire for highthroughput analysis, tomography was not possible; instead to obtain the likely z-positions an energy minimisation was performed. Columns in the starting model were positioned symmetrically about the mid-plane $(\mathrm{z}=0)$. The starting model contained 238 atomic columns with 1656 atoms in total, was 11 atoms high at it thickest, and was assumed to contain no vacancies. The model was then energetically relaxed using a modified Lennard-Jones potential with coefficients reflecting those of platinum. The starting model requires only a few seconds to generate but somewhat longer to relax; the model shown in Figure 2 (left) represents the result after around 31/2 hours. From this 3D atomic model the number of nearest-neighbours can be calculated and used to colour-code the visualisation. A histogram of these coordination numbers (Figure 2, right) then directly indicates the ratios of the various crystallographic 
facets. This ability to observe the relative areas of surface facets opens new possibilities for surface science on an individual particle level and for exploring this in relation to catalytic performance.

\section{References:}

[1] L. L. Chng, N. Erathodiyil, and J. Y. Ying, Accounts of Chemical Research 46 (2013), p. 1825-37.

[2] J. M. Lebeau and S. Stemmer, Ultramicroscopy 108 (2008), p. 1653-8.

[3] L. Jones and P. D. Nellist, Microscopy \& Microanalysis 19 (2013), p. 1050-60.

[4] Software available free of charge for academic / non-commercial use from www.lewysjones.com.

[5] H. E et al, Ultramicroscopy 133 (2013), p. 109-19.

[6] The authors acknowledge funding from the UK-EPSRC and EU-ESTEEM2 programmes.

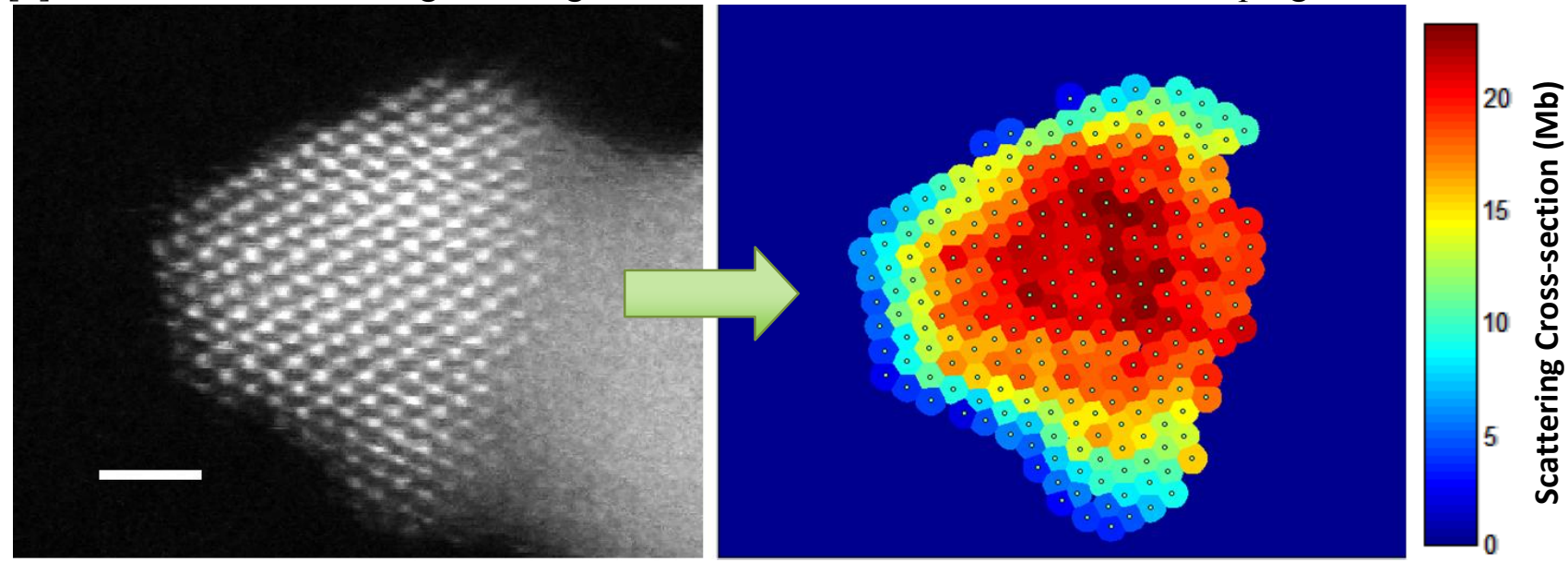

Figure 1. HAADF image of [110] oriented pure platinum nanoparticle (left) and the associated scattering cross-section analysis for each resolvable atomic column (right).
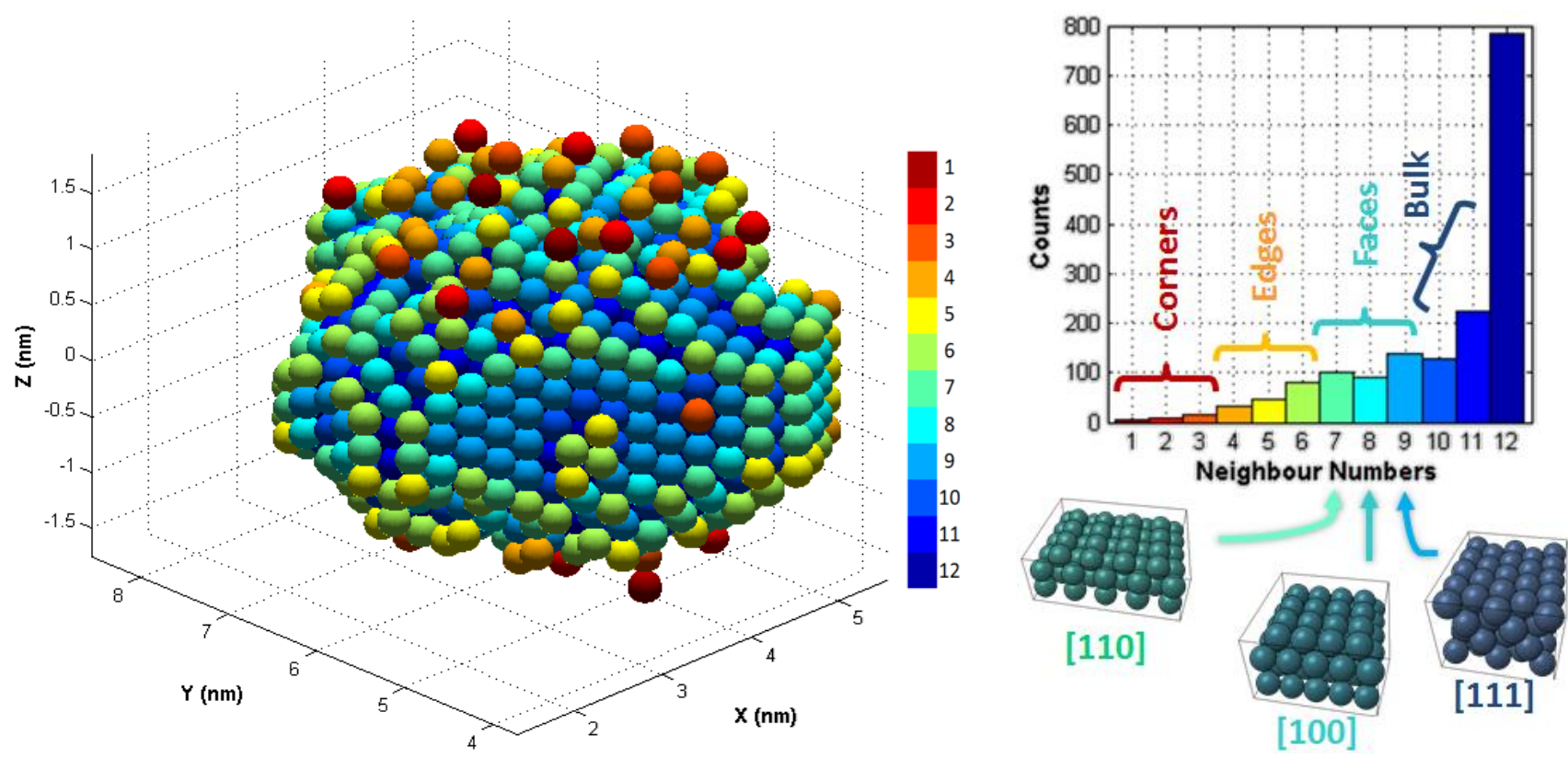

Figure 2. Rebuilt three-dimensional structure of the nanoparticle with colour indicating the nearest-neighbour coordination (left) and accompanying histogram analysis indicating ratios of faceting types. 